\title{
Utilidad de la Proteína Epididimal Humana 4 (HE4) en la detección de Cáncer de ovario.
}

*Correspondencia:

gabriel.moreyl@ug.edu.ec

Teléfono [593] 42282281

Conflicto de intereses: Los autores declaran no tener conflictos de intereses.

Fondos: Ver la página 30

Recibido: 1 Enero 2018

Aceptado: 11 Abril 2018

Publicado: 30 Abril 2018

Membrete bibliográfico:

Núñez-Nájera M, Morey-León G, Nicola-Salas E, Vega Luzuriaga P. Utilidad de la proteína epididimal humana 4 (HE4) en la detección de Cáncer de ovario. Rev. Oncol. Ecu 2018;28(1):22-33

DOI: https://doi.org/10.33821/260

Copyright Núñez-Nájera, et al. Este artículo es distribuido bajo los términos de Creative Commons Attribution License, el cual permite el uso y redistribución citando la fuente y al autor original.

\section{Usefulness of the Human Epididymis protein 4 (HE4) in the detection of ovarian cancer. \\ Mónica Núñez-Nájera1, Gabriel Morey-León* iD, Eva Nicola-Salas³, Patricio Vega-Luzuriaga3.}

1. Laboratorios Núñez, Guayaquil.

2. Universidad de Guayaquil, Guayaquil. Facultad de Ciencias Médicas. Carrera de Obstetricia.

3. Instituto Nacional de Investigación en Salud Pública "Leopoldo Izquieta Pérez", Guayaquil.

\section{Resumen}

Introducción: El cáncer de ovario epitelial aunque tiene baja prevalencia está considerado entre las malignidades ginecológicas más letales por su alta mortalidad. El interés en la detección temprana del cáncer de ovario como mecanismo para lograr la reducción de la mortalidad ha crecido con el descubrimiento de biomarcadores tumorales séricos asociados a tumores malignos. El presente estudio plantea determinar la eficacia del uso del biomarcador HE4 para la detección precoz de cáncer epitelial de ovario en estadios tempranos.

Métodos: Se evaluaron pacientes con masas pélvicas entre abril de 2015 y marzo de 2016 . Valores de sensibilidad, especificidad, predictivo positivo y negativo, razón de probabilidad positiva y negativa, y pruebas estadísticas fueron calculados para determinar la relación entre los estados menopáusicos, y los grupos de acuerdo con el resultado histológico (benigno, maligno y control) de HE4, CA125 y ROMA.

Resultados: Ingresaron al estudio 53 pacientes. La proteína epididimal humana 4 - HE4 presentó un valor medio diferenciable que permite distinguir masas pélvicas malignas (HE4:7,19 (maligno) vs. 5,71 (benigno)), igualmente la combinación HE4 + ROMA presentan mayor sensibilidad y especificidad (S: 100 \%; E: $94.29 \%$ ) que las combinaciones CA125 + HE4 y CA125 + ROMA (S: 80 \% y $88.89 \%$; E: $75.76 \%$ y $77.14 \%)$.

Conclusión: Los resultados sugieren que HE4 serviría como un biomarcador eficiente para la diferenciación de masas pélvicas en estadios tempranos y si se adiciona el estatus menopaúsico, e 
índice ROMA afianzaría los resultados, permitiendo la diferenciación del cáncer de ovario epitelial en estadios tempranos en el país.

Palabras Claves: NEOPLASIAS OVÁRICAS, ANTÍGENO CARCINOEMBRIONARIO, PREMENOPAUSIA, POSMENOPAUSIA.

DOI: $10.33821 / 260$

\section{Abstract}

Introduction: Although epithelial ovarian cancer (EOC) has a low prevalence, it is considered among the most lethal gynecological malignancies due to its high mortality. The interest in the early detection of ovarian cancer as a mechanism to achieve the reduction of mortality has grown with the discovery of serum tumor biomarkers associated with malignant tumors. The present study proposes to determine the efficacy of the use of the HE4 biomarker for the early detection of ovarian epithelial cancer in early stages.

Methods: We evaluated 53 patients with pelvic masses between April 2015 and March 2016. Sensitivity, specificity, positive and negative predictive values, positive and negative likelihood ratio, and statistical tests were calculated to determine the relationship between menopausal states, and groups according to the histological result (benign, malignant and control) of HE4, CA125 and ROMA.

Results: The human epididymal protein 4 - HE4 presented a differentiable mean value that allows to distinguish malignant pelvic masses (HE4: 7.19 (malignant) vs. 5.71 (benign)), likewise the combination HE4 + ROMA present greater sensitivity and specificity (S: 100\%; E: $94.29 \%$ ) than combinations CA125 + HE4 and CA125 + ROMA (S: 80\% and $88.89 \%$; E: $75.76 \%$ and $77.14 \%$ ).

Conclusion: The results suggest that HE4 would serve as an efficient biomarker for the differentiation of pelvic masses in early stages and if menopausal status is added, and ROMA index would strengthen the results, allowing the differentiation of epithelial ovarian cancer in early stages in the country.

Keywords: OVARIAN NEOPLASMS, CARCINOEMBRIONARY ANTIGEN, PREMENOPAUSE, POSMENOPAUSE.

DOI: $10.33821 / 260$

\section{Introducción}

Los tumores de ovario son un grupo de neoplasias diversas por su epidemiología, histogénesis e historia natural [1]. Los tumores epiteliales representan la mayoría de las neoplasias ováricas (más del $90 \%$ ), seguidas de las germinales $(5 \%)$ y finalmente las procedentes del estroma gonadal (4\%). Dentro del grupo de tumores epiteliales existen tumores benignos, borderline o de baja malignidad, y malignos. Los tumores malignos (cancerosos) o de bajo potencial maligno pueden diseminarse (metástasis) a otras partes del cuerpo y pueden ser fatales [2].

El cáncer de ovario epitelial (por sus siglas en Inglés EOC) aunque tiene baja prevalencia es considerado entre las malignidades ginecológicas más letales por su alta mortalidad [3-5], siendo el séptimo cáncer más frecuente diagnosticado mundialmente con 238 mil casos 
nuevos en el 2012 y la octava causa de mortalidad por cáncer con 152 mil muertes [6-8]. A nivel de Ecuador, la tasa de mortalidad reportada según el INEC se registraron 1277 casos de tumores malignos de ovario de los cuales 339 casos eran de mujeres entre los 55 años a 64 años, seguidos de 336 casos entre los 45 a 54 años y 281 casos corresponden a mujeres entre 65 años o más [9].

La etiología del EOC se considera que probablemente sea de carácter multifactorial, sin embargo, se piensa que los factores más asociados a esta enfermedad serían: edad, obesidad, antecedentes relacionados con la reproducción, estatus menopáusico, antecedentes familiares de cáncer de ovario, mama y cáncer colorrectal, entre otros. La mayoría de estos cánceres son encontrados en mujeres posmenopáusicas y sólo el $10-$ $15 \%$ son descubiertos en premenopáusicas. La media de edad para el EOC se sitúa en torno a los 60 años y en menos del $1 \%$ aparecen antes de los 30 años [10].

Debido al incremento del número de mujeres con cáncer de ovario que son referidas a especialistas, rangos de predicción de riesgos han sido desarrollados para identificar mujeres con riesgo alto de cáncer de ovario mediante, la incorporación de datos clínicos (ej. estatus menopaúsico), imagenología (ej. ultrasonido) y/o aplicación de biomarcadores tumorales (HE4, CA125) [11-14]. Diferentes estudios han identificado a la proteína epididimal humana 4-HE4, la cual está presente en altas concentraciones en el epidídimo en hombres y también se encuentra en pacientes con cáncer de ovario, como un marcador para cáncer ovárico con mejorada especificidad en relación con CA125 [15-21].

Con base en lo antes expuesto, la presente investigación tiene como objetivo determinar la utilidad del uso del biomarcador HE4 para la detección precoz de EOC en estadios tempranos en población femenina ecuatoriana, lo cual combinado con chequeos ginecológicos y/o incluyendo el biomarcador CA125 y/o índice ROMA, puedan ser sugeridos como herramienta diagnóstica eficaz que ayude a disminuir y/o controlar eficientemente el avance de este tipo de cáncer que afecta fuertemente a la población.

\section{Materiales y Métodos}

El presente estudio de casos, retrospectivo, observacional se realizó con 53 mujeres en edad fértil que asisten al Hospital de SOLCA de Guayaquil, que presentaron algún tipo de evolución de masas pélvicas, comprendidos entre abril de 2015 y marzo de 2016. La muestra obtenida fue de 53 casos de pacientes que presentaron algún tipo de evolución de masas pélvicas y dolor abdominal al ser ingresadas, en los cuales se encontraban 21 mujeres con masas pélvicas malignas, 12 mujeres con masas pélvicas benignas y 20 de control. Este estudio se realizó siguiendo la normativa ética establecida, considerándose no falsear ni alterar los datos originales, resultados y proceder siempre con honestidad y confidencialidad, luego de recibir la aprobación por la Dirección de Docencia e Investigación del Hospital de SOLCA Guayaquil.

Entre las categorías empleadas en el estudio se encuentran el estatus menopaúsico (premenopáusica y postmenopáusica), edad (premenopáusica $\leq 45$ años y postmenopáusica $\geq 55$ años), tipo de diagnóstico histológico de la masa pélvica (maligna, 
benigna), niveles de los principales biomarcadores tumorales que se emplean para la valoración del riesgo de presentar EOC (HE4, CA125). Se establecieron umbrales de los niveles de los marcadores dependiendo de la edad de las pacientes, teniendo para HE4 (pM/L) en <40 años hasta 60.5; 40-49 años hasta 76.2; 50-59 años hasta 74.3; 60-69 años hasta 82.9 ; >70 años hasta 104 y para CA125: hasta $35.0 \mathrm{U} / \mathrm{ml}$ independiente de la edad.

El cálculo del índice ROMA, fue determinado dependiendo del estatus menopáusico empleando las siguientes fórmulas:

$$
\begin{gathered}
\text { Premenopáusica: } \mathrm{PI}=-12.0+2.38 * \mathrm{LN}[\mathrm{HE} 4]+0.0626 * \mathrm{LN}[\mathrm{CA} 125] \\
\text { Postmenopáusica: } \mathrm{PI}=-8.09+1.04 * \mathrm{LN}[\mathrm{HE} 4]+0.732 * \mathrm{LN}[\mathrm{CA} 125]
\end{gathered}
$$

Considerándose valores del índice ROMA de Alto riesgo de presentar EOC, cuando en paciente premenopáusica los valores están $\geq 11.4$ y en postmenopáusica son $\geq 29$.9; por otro lado, se considera de bajo riesgo de presentar EOC cuando en pacientes premenopáusica los valores están $\leq 11.4$ y en postmenopáusica $\leq 29.9$.

Los valores estadísticos de sensibilidad, especificidad, valor predictivo positivo (VPP), valor predictivo negativo (VPN), razón de probabilidad positiva, razón de probabilidad negativa, de igual manera todos las pruebas estadísticas Coeficiente Correlación Pearson y MannWhitney $U$, para determinar la relación entre los estados menopáusicos, así como los tipos de grupos (benigno, maligno y control) de CA125, HE4 e índice ROMA fueron calculados utilizando el programa MedCalc v 16.2.1(MedCalc Software, Mariakerke, Belgium), para todas las comparaciones estadísticas realizadas. Y el nivel de $P \leq .05$ fue aceptado como estadísticamente significante.

\section{Resultados}

El estudio incluyó 53 pacientes con masas pélvicas, de las cuales 24 (45.28\%) fueron mujeres postmenopáusicas y 29 (54.72\%) mujeres premenopáusicas. Del total, $22.6 \%$ presentaron tumoraciones benignas, $39.6 \%$ tenían EOC y $20,37.8 \%$ correspondían a pacientes control. La edad media de las pacientes con tumores benignos, malignos y control fueron de $38.0 \pm 10.45,55.00 \pm 19.66$ y $46.00 \pm 18.18$ años, respectivamente. El diagnóstico más común en pacientes con malignidad fue Cistoadenocarcinoma seroso (66.67\%).

Los niveles de los biomarcadores tumorales difieren significativamente entre las mujeres con masas benignas y las diagnosticadas con cáncer de ovario, frente al grupo control. Los valores medios de los marcadores HE4 y CA125, así como el índice ROMA de las mujeres diagnosticadas con cáncer de ovario difieren fuertemente frente al control (CA125: $5.72 \pm$ 2.77; HE4: $7.19 \pm 1.57$; ROMA: 2.42), existiendo rangos diferenciales de biomarcador en las mujeres que presentan tumoraciones malignas frente al control (CA125: $1.15 \mathrm{pMol} / \mathrm{L}$; HE4: $1.40 \mathrm{U} / \mathrm{ml}$; ROMA: $5.92 \pm 1.71$ ). En las mujeres con tumoraciones benignas no se encontró diferencia significativa en los niveles de los diferentes biomarcadores e índice evaluado (CA125: 0.07; HE4: -0.08; ROMA: -0.21). Para permitir la diferenciación entre las 
tumoraciones benignas y malignas el índice ROMA es el que mayor diferencial presenta (2.63), seguido de HE4 (1.48) y finalmente CA125 (1.08). De igual manera, HE4 permite identificar mejor las tumoraciones malignas contra índice ROMA y CA125 [7.19 \pm 1.57 $\mathrm{pMol} / \mathrm{L}$ (RIC: $2.47 \mathrm{pMol} / \mathrm{L}$ ) versus $5.92 \pm 1.71$ (RIC: 2.84 ) versus $5.72 \pm 2.77 \mathrm{U} / \mathrm{ml}$ (RIC: 4,88 $\mathrm{U} / \mathrm{ml}$ )] (Tabla 1).

No se observa diferencias significativas entre los grupos estudiados (globales y benigno versus control o maligno versus control) y/o marcador evaluado; sin embargo, se observa diferencia significativa cuando se compara las tumoraciones benignas versus malignas (chi²:4.5371; P. 0.033169) en mujeres premenopáusicas y posmenopáusicas $(P \leq 0.05)$. HE4 difiere significativamente entre las tumoraciones benignas versus malignas $(P .0 .018363)$. No existiendo diferencia significativa entre tumoraciones benignas versus control y malignas versus control; ROMA no presentó diferencia significativa en ninguna de las comparaciones (Tabla 2).

Se observó en el análisis global que HE4 combinado con ROMA presentan mayor sensibilidad y especificidad (S: 100\%; E: $94.29 \%$ ) que las combinaciones CA125 + HE4 y CA125 + ROMA (S: $80 \%$ y 88.89\%; E: $75.76 \%$ y 77.14\%). De igual forma, se mostró el comportamiento en las evaluaciones con relación al estatus menopaúsico, encontrándose que en las mujeres premenopáusicas la combinación HE4 + ROMA muestra mejores valores de los mismos parámetros determinados; en el caso de la posmenopáusicas CA125 + ROMA muestra los mejores resultados seguido por HE4 + ROMA (Tabla 3).

Tabla 1. Estadística descriptiva de los biomarcadores HE4, CA125, ROMA en los grupos de estudio.

\begin{tabular}{|c|c|c|c|c|}
\hline & & Biomarcac & Tumoral & \\
\hline $\begin{array}{l}\text { Tipo de } \\
\text { Tumoración }\end{array}$ & Parámetro & $\mathrm{CA} 125(\mathrm{U} / \mathrm{ml})$ & HE4 pMol/L & ROMA \\
\hline & P25 & 4.28 & 5.44 & 3.06 \\
\hline & Media \pm DS & $4.64 \pm 1.41$ & $5.71 \pm 0.26$ & $3.29 \pm 0.50$ \\
\hline Benigno & P75 & 6.03 & 5.77 & 3.43 \\
\hline$n: 12$ & Max & 7.77 & 6.15 & 4.45 \\
\hline & Min & 3.44 & 5.18 & 2.63 \\
\hline & RIC & 1.76 & 0.32 & 0.37 \\
\hline & P25 & 3.81 & 5.87 & 3.65 \\
\hline & Media \pm DS & $5.72 \pm 2.77$ & $7.19 \pm 1.57$ & $5.92 \pm 1.71$ \\
\hline Maligno & P75 & 8.69 & 8.34 & 6.49 \\
\hline$n .21$ & Max & 12.63 & 10.55 & 6.63 \\
\hline & Min & 2.83 & 5.06 & 1.54 \\
\hline & RIC & 4.88 & 2.47 & 2.84 \\
\hline & P25 & 3,50 & 5.29 & 2.66 \\
\hline & Media \pm DS & $4.57 \pm 1.45$ & $5.79 \pm 0.77$ & $3.50 \pm 1.30$ \\
\hline Control & P75 & 5.45 & 6.17 & 4.37 \\
\hline$n .20$ & Max & 7.70 & 8.12 & 6.31 \\
\hline & Min & 2.71 & 4.97 & 1.38 \\
\hline & RIC & 1.94 & 0.87 & 1.71 \\
\hline
\end{tabular}

DS: desviación Estándar. P25: percentil 25. P75: percentil 75. RIC: Rango intercuartil. 
Tabla 2. Determinación de chi2 y valor $\mathrm{p}$ de los grupos benignos, malignos y control de los diferentes marcadores y estatus menopáusicos.

\begin{tabular}{|c|c|c|c|c|c|c|c|}
\hline & \multicolumn{6}{|c|}{ Tipo de Tumoración } \\
\hline & & \multicolumn{2}{|c|}{ Benigno vs Maligno } & \multicolumn{2}{|c|}{$\begin{array}{l}\text { Benigno vs } \\
\text { Control }\end{array}$} & \multicolumn{2}{|c|}{ Maligno vs Control } \\
\hline $\begin{array}{l}\text { Estatus } \\
\text { Menopaúsico }\end{array}$ & $\mathrm{n}$ & $\mathrm{Chi}^{2}$ & $P$ & $\mathrm{Chi}^{2}$ & $P$ & $\mathrm{Chi}^{2}$ & $P$ \\
\hline Premenopáusicas & 21 & 154 & $0 \cap 3 *$ & חרתח & ? & 106 & 0650 \\
\hline Posmenopáusica & 32 & 4.54 & U.U. & $3.0 \angle 0$ & 0.082 & 0.196 & 0.658 \\
\hline CA125 & & 10.78 & $0.013^{*}$ & 5.294 & 0.151 & 9.504 & $0.023^{*}$ \\
\hline HE4 & & 10.02 & $0.018 *$ & 3.574 & 0.311 & 5.902 & 0.117 \\
\hline ROMA & & 7.46 & 0.059 & 3.959 & 0.266 & 3.456 & 0.327 \\
\hline
\end{tabular}

Para identificar las relaciones existentes entre las variables estudiadas, se probaron el Coeficiente de Correlación Pearson, prueba $U$ de Mann-Whitney, para comparar biomarcadores e índices individuales (CA125, HE4 y ROMA) y las combinaciones CA125 + HE4, CA125 + ROMA y HE4 + ROMA, entre mujeres premenopáusicas y posmenopáusicas. Se pudo observar que el biomarcador HE4 y el índice ROMA, así como la combinación HE4 + ROMA presenta diferencia significativa indiferentemente de la prueba estadística aplicada y el estado menopaúsico de la paciente (Tabla 4).

Tabla 3. Pruebas diagnósticas en pacientes de acuerdo con cada estatus menopáusico por cada combinación de marcadores.

\begin{tabular}{|c|c|c|c|c|}
\hline & & & Estatus menopáus & \\
\hline & Parámetro & $\begin{array}{c}\text { Combinado } \\
n=53\end{array}$ & $\begin{array}{c}\text { Premenopausia } \\
n=29\end{array}$ & $\begin{array}{c}\text { Postmenopausia } \\
n=24\end{array}$ \\
\hline & Sensibilidad & $100 \%$ & $100 \%$ & $100 \%$ \\
\hline & Especificidad & $94.29 \%$ & $100 \%$ & $83.33 \%$ \\
\hline & VPP & $90 \%$ & $100 \%$ & $85.71 \%$ \\
\hline HE4 & VPN & $100 \%$ & $100 \%$ & $100 \%$ \\
\hline & LR+ & 17.50 & ND & 6.00 \\
\hline & LR- & 0.00 & 0.00 & 0.00 \\
\hline & Exactitud & $96.23 \%$ & $100 \%$ & $91.67 \%$ \\
\hline & Sensibilidad & $88.89 \%$ & $66.67 \%$ & $100 \%$ \\
\hline & Especificidad & $77.14 \%$ & $65.22 \%$ & $100 \%$ \\
\hline & VPP & $66.67 \%$ & $33.38 \%$ & $100 \%$ \\
\hline DOILO & VPN & $93,1 \%$ & $88.24 \%$ & $100 \%$ \\
\hline & LR+ & 3,89 & 1.92 & ND \\
\hline & LR- & 0,14 & 0.51 & 0.00 \\
\hline & Exactitud & $81.13 \%$ & $65.52 \%$ & $100 \%$ \\
\hline & Sensibilidad & $80 \%$ & $66.67 \%$ & $85.71 \%$ \\
\hline & Especificidad & $75.76 \%$ & $65.22 \%$ & $100 \%$ \\
\hline & VPP & $66.67 \%$ & $33.38 \%$ & $100 \%$ \\
\hline RE 4 & VPN & $86.21 \%$ & $88.24 \%$ & $83.33 \%$ \\
\hline & LR+ & 3.3 & 1,92 & ND \\
\hline & LR- & 0.26 & 0.51 & 0.14 \\
\hline & Exactitud & $77.36 \%$ & $65.52 \%$ & $91.67 \%$ \\
\hline
\end{tabular}

ND: un valor de la matriz era igual a "0" no determinado permitiendo el cálculo del parámetro. $\mathrm{P} \leq .05$. 
Tabla 4. Pruebas estadísticas por cada combinación de marcadores (CA125, HE4, ROMA) en los diferentes estatus menopáusico y de los marcadores por individual, nivel de confianza de $\mathrm{P} \leq 0.05$.

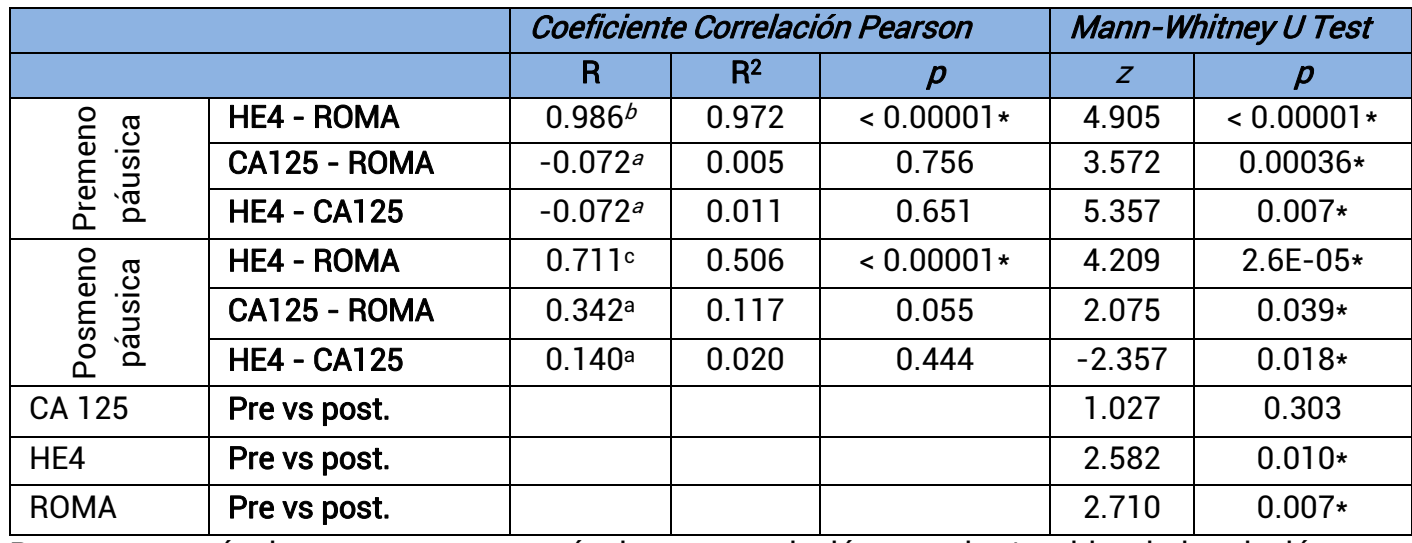

Pre: premenopáusico, post: postmenopáusico. a: correlación negativa/positiva, baja relación entre variables. $b$. correlación positiva, alta relación entre variables. $c$. correlación positiva, moderadamente alta relación entre variables; $*$ : diferencia significativa $p \leq .05$

\section{Discusión}

Esta investigación fue realizada para evaluar la utilidad de HE4 en la detección precoz de cáncer ovárico de células epiteliales comparándolo frente a CA125 e índice ROMA, para los cuales se consideró puntos de corte preestablecidos en cada variable a evaluar. Diferentes estudios sugieren que HE4 es superior a CA125 para distinguir entre enfermedades ováricas malignas y benignas [22-27]. Los resultados muestran que HE4 presenta un valor medio altamente diferenciable, que permite distinguir entre mujeres con masas pélvicas malignas de las benignas ( 7.19 versus 5.71 ; control 5.79 ), al contrario de CA 125 e I. ROMA (5.72 versus 4.64 ; control 4.57 y 5.92 versus 2.29 ; control 3.50 , respectivamente). Estos resultados y los publicados en diferentes estudios concuerdan en relación con la superioridad de HE4 frente a CA125 e I. ROMA solos.

Li et a/[28] y Lin J, Qin J, Sangvatanakul V [29] en sus metaanálisis en el cual se incluyen mujeres con masas pélvicas sospechosas, sugieren que la adición del biomarcador HE4 al algoritmo diagnóstico mejora la especificidad y sensibilidad en la detección de cáncer ovárico. Braicu et a/[30] mostró que ni CA125 ni HE4 serían biomarcadores confiables para el diagnóstico de tumores ováricos bordeline (BOT). La combinación de HE4 con I. ROMA presentó ¿mayor, menor o igual? sensibilidad y especificidad (S: $100 \%$; E: 94.29\%) que las combinaciones CA125 + HE4 y CA125 + ROMA (S: $80 \%$ y $88.89 \%$; E: 75.76 \% y $77.14 \%$ ). Estos resultados confirman los hallazgos obtenidos previamente por otros autores mostrando que la inclusión del biomarcador HE4 al algoritmo, mejoraría la diferenciación del riesgo de cáncer ovárico, demostrando, que la combinación del biomarcador HE4 + I. ROMA asegura significativamente mejores resultados para diferenciar el riesgo de cáncer ovárico, así como diferenciar entre masas benignas y malignas.

Basados en los análisis de correlación de variables, en la cual se determina qué tan relacionados están dos variables para definir un resultado y su exactitud, Anton et al [31] no encontraron diferencias significativas entre mujeres que presentan masas ováricas mediante la medición de CA125, HE4, ROMA, e IRM. Sin embargo, Cho et al [32] evidenciaron que la combinación entre HE4 y ROMA mejoraban la definición de masas pélvicas con un $P<0.001$. Fujiwara et al [33] empleando la prueba $U$ de Mann-Whitney 
mostraron que HE4, CA125 e I. ROMA son biomarcadores valiosos para el diagnóstico de EOC tipo II y que HE4 e I. ROMA mejorarían la diferenciación entre EOC tipo I y masas benignas y la combinación de HE4 y CA125 sería el método más exacto para el diagnóstico de EOC. En nuestros resultados mostramos que empleando la prueba $U$ de Mann-Whitney la mayoría de las combinaciones presentaron diferencias estadísticamente significativas, y al analizarlos individualmente el marcador CA125, fue el que no presentó diferencia significativa (P. 0.30302).

Aunque todos presentan diferencias significativas bajo esta prueba, es la combinación HE4 + ROMA la que presenta una diferencia significativa más representativa (premenopáusica $P<0.00001 *$; posmenopáusica $P .2 .6 \mathrm{E}-05)$; mientras cuando se evalúan los marcadores individuales se observa alta diferencia significativa en HE4 ( $P$. 0.027898) y ROMA ( $P$. 0.004222 ). Los resultados concuerdan con los mostrados por Cho et al [32] y Fujiwara et al [33] en la cual se observa que HE4 y ROMA serían candidatos adecuados para mejorar la diferenciación de masas, y difieren de los de Anton et al [31] en donde no encuentra diferencia entre los marcadores empleados.

Los autores de este estudio, que es uno de los pocos en el país donde se comparan los diferentes biomarcadores tumorales contra el índice ROMA, y se incluye el estatus menopaúsico, para obtener un algoritmo que permita diferenciar los estadios iniciales de cáncer de ovario, infieren según los resultados, que el uso combinado de HE4 + ROMA ó HE4 sólo sería un buen indicador para determinar el riesgo de cáncer ovárico, debido a que ambos comparten similares fortalezas en cuanto a la clasificación de las pacientes más propensas. Este estudio tuvo la limitación de poca población de pacientes analizados. Por lo que, para fortalecer los hallazgos encontrados en el estudio, evaluaciones adicionales deben ser realizadas con estos biomarcadores sobre una población más amplia de pacientes, particularmente de mujeres con EOC.

\section{Conclusiones}

Los resultados obtenidos sugieren que HE4 serviría como un biomarcador eficiente para la diferenciación de masas pélvicas en estadios tempranos y si se adiciona el estatus menopaúsico, y combina con el índice ROMA afianzaría los resultados, permitiendo la diferenciación del EOC en estadios tempranos. En mujeres premenopáusicas la combinación de HE4 e índice ROMA permiten mayor exactitud en la detección de EOC, sin embargo, cuando las mujeres están en estadios posmenopáusicos mejores resultados se obtienen entre CA125 e índice ROMA.

\section{Agradecimientos}

Reconocemos a las personas que participaron indirectamente en el estudio tales como el personal técnico, pacientes y personal de los Laboratorios Núñez, de la Universidad de Guayaquil, Facultad de Ciencias Médicas-Carrera de Obstetricia y del Instituto Nacional de Investigación en Salud Pública "Leopoldo Izquieta Pérez", Guayaquil. 


\section{Información adicional}

Nota del Editor

La Revista Oncología Ecu permanece neutral con respecto a los reclamos jurisdiccionales en mapas publicados y afiliaciones institucionales.
Abreviaturas

EOC: Siglas en inglés del Cáncer de Ovario Epitelial.

HE4: proteína epididimal Humana 4.

ROMA: Siglas en inglés de "Risk of Ovarian Malignancy Algorithm". Riesgo de malignidad ovárica, algoritmo.

Archivos Adicionales

Ninguno declarado por los autores.

\section{Fondos}

Los fondos de la investigación fueron propios de los autores del presente artículo.

\section{Disponibilidad de datos y materiales}

Existe la disponibilidad de datos bajo solicitud al autor de correspondencia. No se reportan otros materiales.

\section{Contribuciones de los autores}

MNN, GML, PVL, realizaron la idea de investigación, revisión bibliográfica. ENS, recolección de datos, escritura del artículo. PVL y MNN realizaron el análisis crítico del artículo. ENS realizó las correcciones editoriales. Todos los autores leyeron y aprobaron la versión final del artículo.

Aprobación de ética y consentimiento para participar

No aplica a este estudio.

\section{Consentimiento para publicación}

No aplica. 
Información de los autores

Mónica Núñez-Nájera, M.Sc., Laboratorios Núñez, Guayaquil.

Gabriel Morey-León, M.Sc., Universidad de Guayaquil, Guayaquil. Carrera de Obstetricia. Facultad de Ciencias Médicas. iD https://orcid.org/0000-0003-1824-8285

Eva Nicola-Salas, M.Sc., Instituto Nacional de Investigación en Salud Pública "Leopoldo Izquieta Pérez", Guayaquil.

Patricio Vega-Luzuriaga, M.Sc., Instituto Nacional de Investigación en Salud Pública "Leopoldo Izquieta Pérez", Guayaquil.

Revisiones por pares

Acceda a la revisión de pares académicos en el siguiente enlace: https://publons.com/review/3858042

\section{Referencias}

Abreviaturas en la referencias DOI: Digital Object Identifier

PMID: PubMed Identifier SU: Short URL
1. DiSaia PJ, Creasman WT, Mannel RS, McMeekin DS, Mutch DG. Clinical Gynecologic Oncology E-Book. Elsevier Health Sciences. 2017. ISBN: 9780323443173

2. Howell VM, Davidson B. Advances in Epithelial Ovarian Cancer: Model Systems, Microenvironmental Influences, Therapy, and Origins. Frontiers in oncology 2015;5:205. DOI: 10.3389/fonc.2015.00205

3. Eo WK, Kim KH, Park EJ, Kim HY, Kim HB, Koh H, et.al. Diagnostic accuracy of inflammatory markers for distinguishing malignant and benign ovarian masses. Journal of Cancer. 2018:9(7);1165. DOI: 10.7150/jca.23606

4. Wilailak S, Chan KK, Chen $\mathrm{CA}$, Nam JH, Ochiai K, Aw TC, et al. Distinguishing benign from malignant pelvic mass utilizing an algorithm with HE4, menopausal status, and ultrasound findings. Journal of gynecologic oncology. 2015;26(1):46-53. DOI: 10.3802/jgo.2015.26.1.46

5. Torre LA, Islami F, Siegel RL, Ward EM, Jemal A. Global cancer in women: burden and trends. 2017;26(4):444-457. DOI: 10.1158/1055-9965.

6. Bray F, Ferlay J, Soerjomataram I, Siegel RL, Torre LA, Jemal A. Global cancer statistics 2018: GLOBOCAN estimates of incidence and mortality worldwide for 36 cancers in 185 countries. CA: a cancer journal for clinicians. 2018;68(6):394-424. DOI: 10.3322/caac.21492

7. Jemal A, Siegel R, Ward E, Hao Y, Xu J, Thun MJ. Cancer statistics, 2009. CA: A Cancer Journal for Clinicians 2009;59(4): 225-249. DOI: 10.3322/caac.20006

8. American Cancer Society. Cancer Facts \& Figures 2016. Atlanta: American Cancer Society; 2016. SU: bit.ly/2svdIFX

9. Instituto Nacional de Estadísticas y Censos. Anuario de Estadísticas Hospitalarias Camas y Egresos 2013. Quito. 2013.

10. La Vecchia C. Ovarian cancer: epidemiology and risk factors. European journal of cancer prevention. 2017;26(1): 55-62. SU: bit.ly/2M63clb 
11. Abraham J. OVA1 test for preoperative assessment of ovarian cancer. Community Oncology. 2010;6(7):249-250. DOI: 10.1016/S1548-5315(11)70565-4

12. Jacobs I, Oram D, Fairbanks J, Turner J, Frost C, Grudzinskas JG. A risk of malignancy index incorporating CA 125, ultrasound and menopausal status for the accurate preoperative diagnosis of ovarian cancer. BJOG: An International Journal of Obstetrics \& Gynaecology, 1990;97(10):922-929. SU: bit.ly/2HbRs8d

13. Moore R, Brown A, Miller M, Skates S, Allard W, Verch T, et al. The use of multiple novel tumor biomarkers for the detection of ovarian carcinoma in patients with a pelvic mass. Gynecologic oncology. 2008;108(2):402-408. SU: bit.ly/2Fz7iHD

14. Nunes N, Yazbek J, Ambler G, Hoo W, Naftalin J, Jurkovic D. Prospective evaluation of the IOTA logistic regression model LR2 for the diagnosis of ovarian cancer. Ultrasound in Obstetrics \& Gynecology. 2012;40(3):355-359. DOI: 10.1002/uog. 11088.

15. Yang WL, Lu Z, Lu KH, Menon U, Kobayashi M, Hanash S, et al Abstract LB-176: HE4 antigenautoantibody complexes complement $\mathrm{CA} 125$ for detecting early stage ovarian cancer and can be elevated with CA125 in preclinical ovarian cancer. 2018.

16. LaVigne K, Dao F, Abu-Rustum N, Jelinic P, Ramanathan LV, Fleisher M, et al. Abstract AP01: HE4 is a biomarker for newly diagnosed and recurrent high-grade serous ovarian cancers with normal ca-125 values. 2017.

17. Blackman A, Miller M, Eklund E, Strongin RM, Messerlian G, Moore RG. The utility of additional ovarian cancer biomarkers to the dual marker combination of HE4 and CA-125 for the detection of cancer. Gynecologic Oncology. 2018;149:67-68. DOI: 10.1016/j.ygyno.2018.04.153

18. Mitchell J, Miller M, Singh R, Messerlian G, Moore R. The differential analysis of serum HE4 levels in epithelial ovarian cancer and other malignant tumors. Gynecologic Oncology. 2017;145:88. DOI: 10.1016/j.ygyno.2017.03.206

19. Bairati I, Gregoire J, Plante M, Douville P. Performance of preoperative plasma HE4 and CA- 125 levels in predicting ovarian cancer mortality in women with epithelial ovarian cancer (EOC). Journal of Clinical Oncology 2017;35:15. DOI: 10.1200/JC0.2017.35.15_suppl.e17076

20. Scaletta G, Plotti F, Luvero D, Capriglione S, Montera R, Miranda A, et al. The role of novel biomarker HE4 in the diagnosis, prognosis and follow-up of ovarian cancer: a systematic review. Expert review of anticancer therapy. 2017;17(9):827-839. DOI: 10.1080/14737140.2017.1360138.

21. Braicu El, Torsten U, Richter R, Beteta C, Boneß-Zaloume J, Dimitrova D, et al. HE4 is the marker of choice in discriminating endometriosis from ovarian cancer in pelvic mass patients: Sub-analysis of a prospective multicentric study. Gynecologic Oncology. 2018;149:242. DOI: 10.1016/j.ygyno.2018.04.547

22. Kondalsamy-Chennakesavan S, Hackethal A, Bowtell D, Obermair A, Australian Ovarian Cancer Study Group. Differentiating stage 1 epithelial ovarian cancer from benign ovarian tumours using a combination of tumour markers HE4, CA125, and CEA and patient's age. Gynecologic oncology. 2013;129(3):467-471. DOI: 10.1016/j.ygyno.2013.03.001

23. Kil K, Chung JE, Pak HJ, Jeung IC, Kim J, Jo H. Usefulness of CA125 in the differential diagnosis of uterine adenomyosis and myoma. European Journal of Obstetrics \& Gynecology and Reproductive Biology. 2015;185:131-135. DOI: 10.1016/j.ejogrb.2014.12.008.

24. Desbéne C, Maiga R, Gaillard O. Immunoanalytical characteristics of HE4 protein. In Annales de biologie Clinique. 2018;76(2);225-233. DOI: 10.1684/abc.2018.1330.

25. Lee S, Choi S, Lee Y, Chung D, Hong S, Park N. Role of human epididymis protein 4 in chemoresistance and prognosis of epithelial ovarian cancer. Journal of Obstetrics and Gynaecology Research. 2017;43(1):220-227. DOI: 10.1111/jog.13181. 
26. Sandow J, Rainczuk A, Infusini G, Makanji M, Bilandzic M, Wilson A, et al. Discovery and validation of novel protein biomarkers in ovarian cancer patient urine. PROTEOMICS-Clinical Applications. 2018;12(3):1700135. DOI: 10.1002/prca.201700135.

27. Jia M, Deng J, Cheng X, Yan Z, Li Q, Xing Y, et al. Diagnostic accuracy of urine HE4 in patients with ovarian cancer: a meta-analysis. Oncotarget. 2017;8(6):9660. DOI: 10.18632/oncotarget.14173.

28. Li F, Tie R, Chang K, Wang F, Deng S, Lu W, et al. Does risk for ovarian malignancy algorithm excel human epididymis protein 4 and $\mathrm{CA} 125$ in predicting epithelial ovarian cancer: a meta-analysis. BMC cancer. 2012;12(1):258. DOI: 10.1186/1471-2407-12-258.

29. Lin J, Qin J, Sangvatanakul V. Human epididymis protein 4 for differential diagnosis between benign gynecologic disease and ovarian cancer: a systematic review and meta-analysis. European Journal of Obstetrics \& Gynecology and Reproductive Biology. 2013;167(1): 81-85. DOI: 10.1016/j.ejogrb.2012.10.036.

30. Braicu El, Van Gorp T, Nassir M, Richter R, Chekerov R, Gasimli K, et al. Preoperative HE4 and ROMA values do not improve the CA125 diagnostic value for borderline tumors of the ovary (BOT)-a study of the TOC Consortium. Journal of ovarian research. 2014;7(1):49. DOI: $\underline{10.1186 / 1757-2215-7-49}$

31. Anton C, Carvalho F, Oliveira E, Maciel G, Baracat E, Carvalho J. A comparison of CA125, HE4, risk ovarian malignancy algorithm (ROMA), and risk malignancy index (RMI) for the classification of ovarian masses. Clinics. 2012;67(5): 437-441.

32. Cho H, Park S, Park Y, Kim H, Kang J, Hong S, et al. Comparison of HE4, CA125, and risk of ovarian malignancy algorithm in the prediction of ovarian cancer in Korean women. Journal of Korean medical science. 2015;30(12):1777-1783. DOI: 10.3346/jkms.2015.30.12.1777.

33. Fujiwara H, Suzuki M, Takeshima N, Takizawa K, Kimura E, Nakanishi T, et al. Evaluation of human epididymis protein 4 (HE4) and Risk of Ovarian Malignancy Algorithm (ROMA) as diagnostic tools of type I and type II epithelial ovarian cancer in Japanese women. Tumor Biology. 2015;36(2):1045-1053. DOI: $10.1007 / \mathrm{s} 13277-014-2738-7$ 